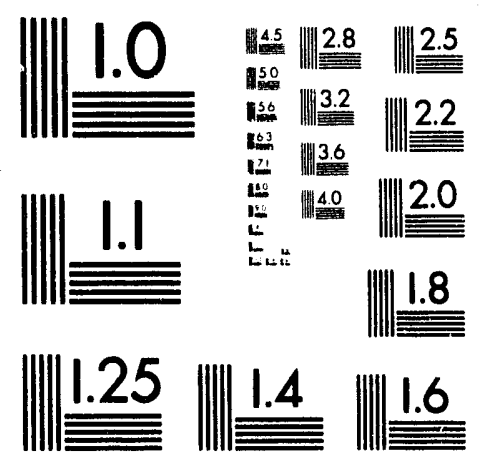



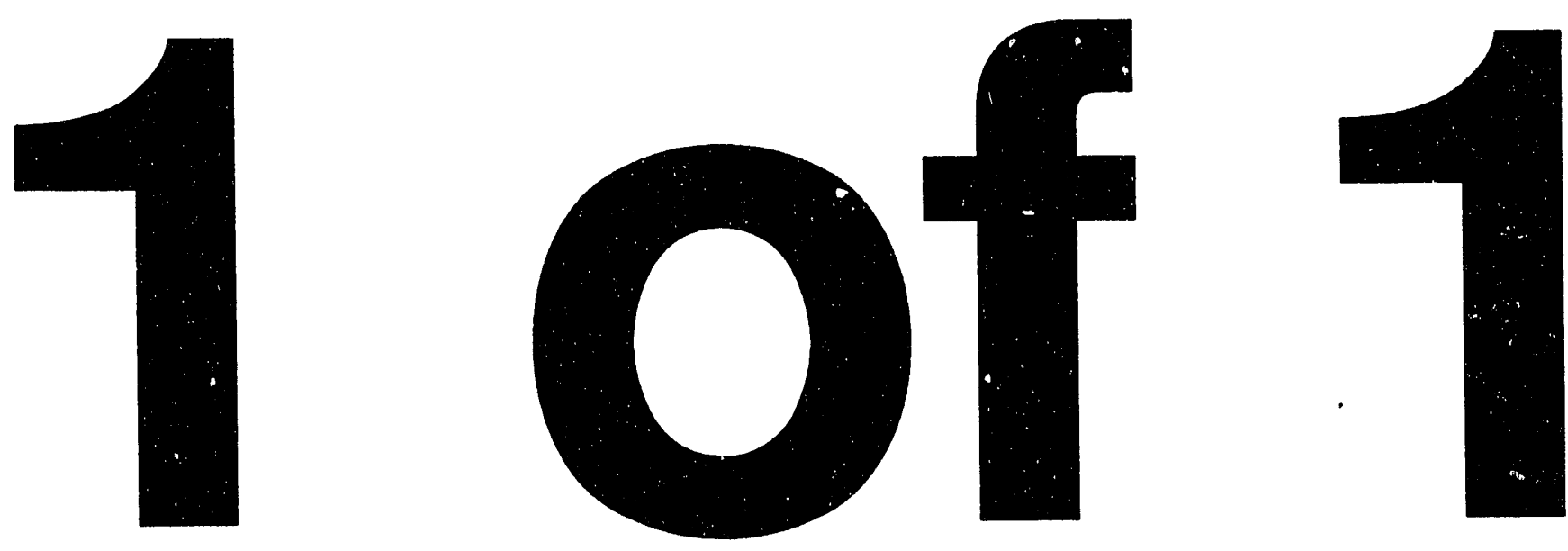


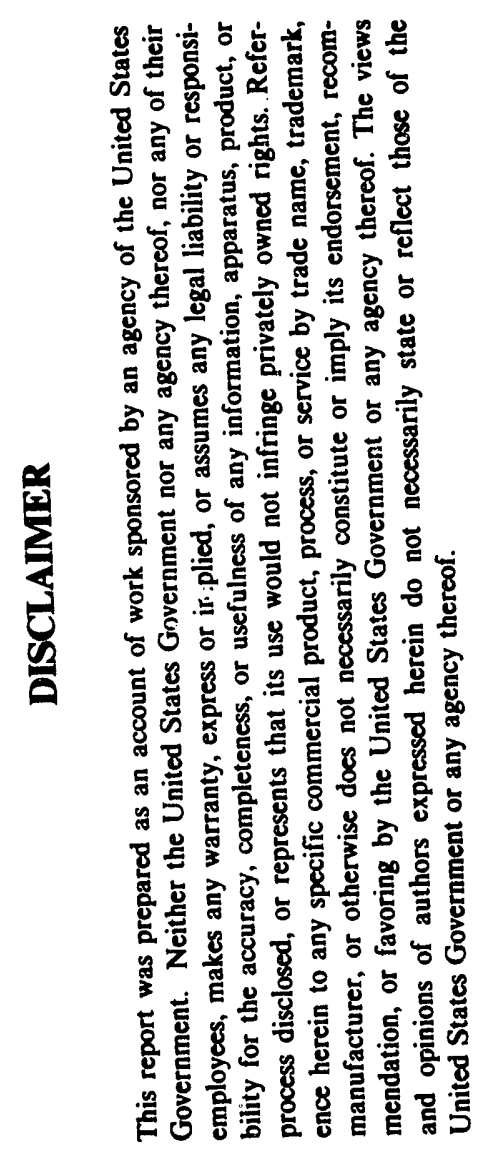

\author{
SYSTEMS OPTIMIZATION LABORATORY \\ DEPARTMENT OF OPERATIONS RESEARCH \\ STANFORD UNIVERSITY \\ STANFORD, CALIFORNIA 94305-4022
}

\title{
The Simplex Algorithm with a New Primal and Dual Pivot Rule
}

by

Hsin-Der Chen ${ }^{*}$, Panos M. Pardaios** and Michael A. Saunders ${ }^{\dagger}$

TECHNICAL REPORT SOL 93-5

June 1993

\begin{abstract}
-Department of Industrial and Systems Engineering, University of Florida, Gainesville, FL 32611. Partially supported by National Science Foundation Grant DDM-8814075.

**Department of Industrial and Systems Engineering, University of Florida, Gainesville, FL 32611. Partially supported by DIMACS under National Science Foundation Contract STC-91-19999.

†Systems Optimization Laboratory, Department of Operations Research, Stanford University, Stanford, CA 94305-4022. Partially supported by Department of Energy Grant DEFG03-92ER25117, National Science Foundation Grant DDM-9204208, and Office of Naval Research Grant N00014-90-J-1242.

Any opinions, findings, and conclusions or recommendations expressed in this publication are those of the authors and do not necessarily reflect the views of the above sponsors.

Also listed as Operations Research Department Technical Report 93-10. Reproduction in whole or in part is permitted for any purposes of the United States Government. This document has been approved for public release and sale; its distribution is unlimited.
\end{abstract}




\title{
The Simplex Algorithm with a New Primal and Dual Pivot Rule
}

\author{
Hsin-Der CHEN, Panos M. PARDALOS* and Michael A. SAUNDERS ${ }^{\dagger}$ \\ Technical Report SOL 93-5 \\ June 1993
}

\begin{abstract}
We present a simplex-type algorithm for linear programming that works with primal-feasible and dual-feasible points associated with bases that differ by only one column.
\end{abstract}

\section{Introduction}

Consider the following linear programming problem:

$$
\text { (P) } \max \left\{c^{T} x \mid A x=b, x \geq 0\right\},
$$

where $A \in R^{m \times n}(m \leq n)$ and the vectors $b, c, x$ have appropriate dimension. Let $B$ be a basis in $A$, so that $A Q=(B N)$ for some permutation $Q$, and let $B x_{B}=b$, $B^{T_{\pi_{B}}}=c_{B}$ and $r=c-A^{T_{\pi}}$. An iteration of the primal simplex method [Dan63] can be briefly described as:

Given $B$ such that $x_{B} \geq 0$, if $r \leq 0$, then stop; otherwise, update $B$.

The dual simplex method is:

Given $B$ such that $r \leq 0$, if $x_{B} \geq 0$, then stop; otherwise, update $B$.

The solution in both cases is given by $x_{B}$ and $x_{N}=0$.

\subsection{The Aim}

We propose an algorithm that maintains two basic solutions for $(P)$. One is primal feasible and the othe: dual feasible, and the bases differ by only one column. Some preliminary computational results have been obtained (see Section 8), showing promise for the algorithm relative to the primal simplex method.

\footnotetext{
"Department of Industrial and Systems Engineering, University of Florida, Gainesville, FL 32611. The first author was partially supported by the National Science Foundation Grant DDM8814075; the second author was partially supported by DIMACS (a National Science Foundation Science and Technology Center, funded under contract STC-91-19999).

'Systems Optimization Laboratory, Department of Operations Research, Stanford University, Stanford, CA 94305-4022. Partially supported by Department of Energy Grant DE-FG0392ER25117, National Science Foundation Grant DDM-9204208, and Office of Naval Research Grant N00014-90-J-1242.
} 


\subsection{Related Work}

Many pivot rules are known for simplex-type algorithms. For example, a large number are described in [TZ92]. Under certain circumstances the proposed algorithm is equivalent to Lemke's method [Lem65], as discussed in Section 6, but otherwise the algorithm appears to be new.

\section{The Main Feature}

For problem $(P)$, let $B_{P}$ and $B_{D}$ be bases in the primal and dual simplex methods respectively. Consider the special case in which $B_{P}$ and $B_{D}$ differ in exactly one column. Let $d$ in $B_{D}$ be the column of $A$ that distinguishes $B_{P}$ and $B_{D}$. Also let $x_{P}$ and $x_{D}$ be the associated primal-feasible and dual-feasible vertices of $(P)$.

Theorem 2.1. If $x_{P}$ and $x_{D}$ are not both optimal vertices, then either $d$ can enter $B_{P}$ in the next primal pivot, or $d$ can leave $B_{D}$ in the next dual pivot.

Proof. Consider a modified problem $\left(P^{\prime}\right)$ in which all variables that are in neither the primal basis nor the dual basis are fixed at zero. It is easy to see that $x_{P}$ and $x_{D}$ are also primal-feasible and dual-feasible vertic $s$ of $\left(P^{\prime}\right)$. In this case, the variable corresponding to $d$ is the only nonbasic variable (for $B_{P}$ ).

By definition of optimality, if $x_{P}$ is not an optimal vertex for $\left(P^{\prime}\right), d$ can enter $B_{P}$ in order to improve the primal objective value. Otherwise, if $x_{D}$ is not an optimal vertex for $\left(P^{\prime}\right), d$ can leave $B_{D}$ in order to improve the dual objective value.

Since all the data $A, b, c, B_{P}$ and $B_{D}$ are the same in $\left(P^{\prime}\right)$ and $(P)$, the result also applies to $(P)$.

Initialization is discussed later. To show that the algorithm continues, we must show that the new bases differ by one column in the next iteration.

Let $\bar{d}$ in $B_{P}$ be the column that is not in $B_{D}$. According to the theorem, $d$ may enter $B_{P}$ in a primal pivot. If the leaving column is $\bar{d}$, then $B_{P}$ and $B_{D}$ are identical and the optimal vertex is reached; otherwise, $B_{P}$ still differs from $B_{D}$ in one column $\left(\bar{d}\right.$ in $B_{P}$ and the leaving column in $\left.B_{D}\right)$.

On the other hand, $d$ may leave $B_{D}$ in a dual pivot. In this case, if the entering column is $\bar{d}$, then we are done; otherwise, $B_{P}$ and $B_{D}$ still have $m-1$ columns in common.

The algorithm therefore proceeds in this way: the leaving column in a primal pivot is a candidate leaving column in the next dual pivot, and the entering column in a dual pivot is a candidate entering column in the next primal pivot.

\section{Remarks}

- There is no pricing step in the simplex algorithm with this pivot rule. The algorithm may be interpreted as a primal simplex method that uses a related dual simplex procedure to do pricing. 
- Since $B_{D}^{-1}$ is closely related to $B_{P}^{-1}$, we don't have to keep both of them. (We can maintain a factorization of just $B_{P}$.) Moreover, since there is no pricing step, we don't have to compute shadow prices $(\pi)$ in the primal method. Similarly, we don't have to compute $B_{D}^{-1} b$ in the dual method. Therefore, the computational effort is the same as in the ordinary simplex method.

- Suppose tinat $\left(x_{P}, x_{D}\right)$ is moved to $\left(x_{P}^{\prime}, x_{D}^{\prime}\right)$ after an iteration (and both $B_{P}$ and $B_{D}$ are upciated). If the primal step is degenerate, then $x_{P}^{\prime}=x_{P}$; otherwise $c^{T} x_{P}^{\prime}>c^{T} x_{P}$. If the dual step is degenerate, then $c^{T} x_{D}^{\prime}=c^{T} x_{D}$; otherwise $c^{T} x_{D}^{\prime}<c^{T} x_{D}$. Therefore, the duality gap will strictly decrease; that is, $c^{T} x_{D}^{\prime}-$ $c^{T} x_{P}^{\prime}<c^{T} x_{D}-c^{T} x_{P}$, except in the very special case where both primal and dual degeneracies exist simultaneously.

- If $B_{P}^{-1}$ or $B_{P}^{-1} A$ is sparse, as for example in network problems, the algorithm needs to compute only part of $B_{P}^{-1} b$. Also, since there is no pricing step, only some of the shadow prices have to be computed (to determine the entering column in the dual simplex step).

In contrast, with minimum-cost network flow problems (for example), although only the flow values in the augmented path need to be updated at each iteration, conventional methods have to compute all the shadow prices (except with partial pricing).

\section{An Example}

Figure 1 provides a two-dimensional example. The superscript indicates the order in which vertices are visited in the algorithm: $x_{P}^{1} \rightarrow x_{D}^{2} \rightarrow x_{P}^{3} \rightarrow x_{D}^{4} \rightarrow x_{P}^{5} \rightarrow x_{D}^{6} \rightarrow x^{*}$.

Note that the algorithm updates both primal-feasible and dual-feasible solutions at each iteration. Therefore, it takes three iterations to solve the example. In fact, any primal simplex method starting from $x_{P}^{1}$ will solve this example in exactly three iterations.

\section{A Variation}

A vertex is adjacent to another vertex if the associated bases differ by one column. Here we suggest a possible variation of the algorithm:

Given a primal-feasible vertex, move to the adjacent dual-feasible vertex with the minimal dual objective value. Then move to the best adjacent primal-feasible vertex, and so on.

The path of this variation algorithm in the example will be $x_{P}^{1} \rightarrow x^{\prime} \rightarrow x^{*}$.

To implement the variation, we must be able to find the appropriate adjacent vertices. Suppose that $B$ is a basis for $(P)$. Let $\hat{A}, x_{B}, \pi$ and $r$ be defined by $B \hat{A}=A$, $B x_{B}=b, B^{T} \pi=c_{B}$ and $r=c-A^{T_{\pi}}$. Let $S_{+}=\left\{j \mid j\right.$ is nonbasic and $\left.r_{j}>0\right\}$ and $S_{-}=\left\{j \mid j\right.$ is nonbasic and $\left.r_{j} \leq 0\right\}$. If there exists some nonbasic variable $j$ with 


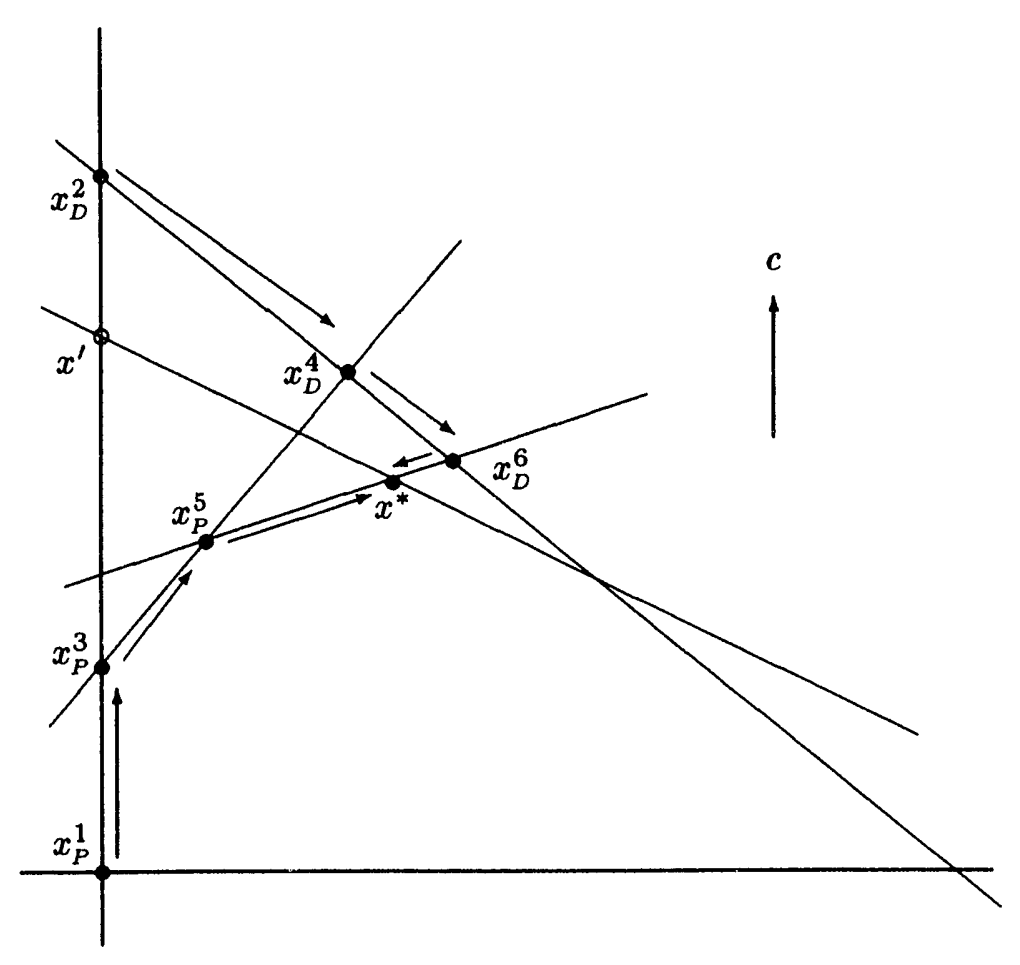

Figure 1: A two-dimensional example.

$r_{j}<0$, then for some basic variable $i$, we r:ay obtain a dual-feasible vertex by deleting variable $i$ from $B$ if the following case holds:

Case 1: $\hat{A}_{i j}>0 \forall j \in S_{+}$, and

$$
\max _{j}\left\{\frac{r_{j}}{\hat{A}_{i j}} \mid j \in S_{+}\right\} \leq \min _{j}\left\{\frac{r_{j}}{\hat{A}_{i j}} \mid j \in S_{-}, \hat{A}_{i j}<0\right\} .
$$

Let $R_{+}=\left\{i \mid i\right.$ is basic and $\left.\left(x_{B}\right)_{i}>0\right\}$ and $R_{-}=\left\{i \mid i\right.$ is basic and $\left.\left(x_{B}\right)_{i} \leq 0\right\}$. Similarly, if there exists some basic variable $i$ with $\left(x_{B}\right)_{i}<0$, then for some nonbasic variable $j$, we may obtain a primal-feasible vertex by adding variable $j$ to $B$ if the following case holds:

Case 2: $\quad \hat{A}_{i j}<0 \forall i \in R_{+}$, and

$$
\max _{i}\left\{\frac{\left(x_{B}\right)_{i}}{\hat{A}_{i j}} \mid i \in R_{-}\right\} \leq \min _{i}\left\{\frac{\left(x_{B}\right)_{i}}{\hat{A}_{i j}} \mid i \in R_{+}, \hat{A}_{i j}>0\right\} \text {. }
$$

\section{Initialization}

Now it is time to say something about initialization. Suppose that a nonsingular basis $B$ of $(P)$ is given. If the initial vertex is not primal feasible $\left(x_{B} \nsucceq 0\right)$, then we may add an artificial variable (column), say $j$, such that Case 2 is satisfied. For 
example, $A_{i j}=-1$ for all $i \in R_{-}$, and $A_{i j}=0$ otherwise; and $c_{j}$ is assigned a large negative value. Then a primal-feasible vertex can be obtained by choosing this artificial variable to enter the basis.

A dual-feasible vertex can be obtained in a similar way by adding a redundant constraint that satisfies Case 1 (now we have $B_{P}$ ) and deleting the corresponding slack variable. The new basis is $B_{D}$.

\section{Relationship to Lemke's Method}

There is a similarity between the new algorithm and Lemke's [Lem65]. If the initial nonsingular basis $B$ corresponds to a primal-feasible vertex of $(P)$, then both algorithms are exactly the same (they follow the same path from that vertex to the solution). The two algorithms differ in the following aspects:

- Lemke's method works on a larger dimension matrix (involving $A$ and $A^{T}$ ).

- When $B$ does not correspond to a primal feasible vertex of $(P)$ the new algorithm uses two artificial variables instead of one as in Lemke's.

- In Lemke's method, the primal and dual variable pairs are "almost complementary"; i.e, they are complementary except for one pair of variables, both of which are nonbasic. In the new algorithm, there are two pairs of variables that are not complementary. (In one pair, both variables are basic; in the other pair, both are nonbasic.)

- There is only one path to the optimal solution in Lemke's method, but the new algorithm is flexible (for example the variation mentioned above).

\section{Summary of the Algorithm}

We now summarize the algorithm. We assume that a nonsingular basis $B_{P}$ is given.

1. Solve $B_{P} x_{B}=b$.

2. If $B_{P}$ is not primal feasible $\left(x_{B} \geq 0\right)$, find another initial $B_{P}$.

(a) Add $a_{P}=-B e$ to $A$ as an artificial column, where $e$ is a columns of 1 s. Set the corresponding entry in $c$ to be $-M$, for some large number $M$.

(b) Remove the column corresponding to the most negative $x_{B}$ from $B_{P}$.

(c) Add the artificial column to $B_{P}$. Solve $B_{P} x_{B}=b$.

3. Solve $B_{P}^{T} \pi=c_{B}$ and compute reduced costs $r=c-A^{T} \pi$. Define $B_{D}=B_{P}$.

4. If $B_{D}$ is dual feasible $(r \leq 0)$, then either $x_{B}$ and $x_{N}=0$ form an optimal solution (if no artificial column was added in Step 2), or the problem is infeasible; stop. Otherwise, find another initial $B_{D}$.

(a) Add an artificial row to $A$ with zeros in basic columns and ones in nonbasic columns. Set the corresponding entry in $b$ to be $\infty$. 
(b) Add a new artificial column $a_{D}=(0, \ldots, 0,1)^{T}$ to $A$. Set the corresponding entry in $c$ to be 0 .

(c) Expand $B_{P}$ by adding this new column and the appropriate part of the artificial row.

(d) Solve $B_{P}^{T} \pi=c_{B}$ and compute reduced $\operatorname{costs} r=c-A^{T} \pi$.

(e) Let $d$ be the column with the most positive element of $r$.

(f) Let $B_{D}=B_{P}$ except that $a_{D}$ is replaced by $d$.

5. Repeat until $d=a_{D}$.

(a) Solve $B_{P} x_{B}=b$ and $B_{P} y=d$.

(b) Determine the index $p=\arg \min \left\{\left(x_{B}\right)_{i} / y_{i} \mid y_{i}>0\right\}$.

(c) Let the $p$ th column of $B_{P}$ be $a_{l}$.

(d) If $a_{l}=a_{D}$, the problem is unbounded; stop.

(e) Let $B_{D}=B_{P}$ except that $a_{D}$ is replaced by $d$.

(f) Solve $B_{D}^{T} \pi=c_{B}$ and $B_{D}^{T} v=e_{p}$.

(g) Compute $r=c-A^{T} \pi$ and $w=A^{T} v$ (the $p$ th row of $B_{D}^{-1} A$ ).

(h) Determine the index $s=\arg \min \left\{r_{j} / w_{j} \mid w_{j}<0\right\}$.

(i) Replace column $a_{l}$ by $d$ in $B_{P}$.

(j) Let $d=a_{s}$.

6. If $a_{P}$ is in $B_{P}$, the problem is infeasible; stop.

7. Otherwise, find the optimal solution.

(a) Remove $a_{D}$ and the corresponding row from $B_{P}$.

(b) Solve $B_{P} x_{B}=b$ and set $x_{N}=0$.

In practice, we could use $y$ in $5(\mathrm{a})$ to update $x_{B}$, and $w$ in $5(\mathrm{~g})$ to update $r$. The main work per iteration is therefore solving $B_{P} y=d$ and $B_{D}^{T} v=e_{p}$ and forming $w=A^{T} v$. (Only nonbasic entries are needed.) This is analogous to one iteration of the primal simplex method with full pricing.

Two forms of partial pricing are also possible. First, columns with the most negative $r_{j}$ could be temporarily ignored (keeping $x_{j}=0$ ). The bases $B_{P}$ will remain primal feasible. When the columns are reconsidered, we have to check that the current basis $B_{D}$ is dual feasible. If so, the algorithm continues; otherwise, a new dual-feasible vertex must be constructed as in 4(a)-4(f).

Alternatively, rows with the most positive $\left(x_{B}\right)_{i}$ could be temporarily ignored. When they are reconsidered, we have to check that $B_{P}$ is primal feasible. If not, a new vertex must be constructed as in $2(\mathrm{a})-2(\mathrm{c})$. 


\section{Experimental Results}

The algorithms (original and variational) have been coded in MATLAB [MLB87] and compared with the simplex method with least-reduced-cost pivot rule (also coded in MATLAB). Full pricing was used in all methods. Some random test problems were generated with the following properties. All constraints (except for non-negativity) are tangent to a unit ball. None of the constraints is redundant. The center of the unit ball is either at the origin or at a point within two units of the origin.

The initial vertex was taken to be the origin. The position of the center therefore determines whether the initial vertex is (primal) feasible or not.

Table 1 lists results for 20 test protlems with varying dimensions and center. The ite ation numbers for the three algorithms are shown for each problem. Note that fo: least-reduced-cost simplex method (LP) and our original algorithm (PDO), the iteration number is the number of primal vertices visited. For our variational algorithm (PDV), it is the number of primal and dual vertices visited. Hence, the iteration numbers reflect the relative computation times for the three algorithms.

\begin{tabular}{|rrl|rrr|rrr|}
\hline$m$ & $n$ & density & \multicolumn{2}{|c|}{$\begin{array}{c}\text { LP } \\
\text { Origin feasible }\end{array}$} & \multicolumn{2}{|c|}{$\begin{array}{c}\text { PP } \\
\text { Origin infeasible }\end{array}$} \\
\hline 50 & 25 & 0.3 & 38 & 37 & 63 & 36 & 26 & 49 \\
100 & 25 & 0.3 & 41 & 42 & 66 & 32 & 26 & 41 \\
150 & 25 & 0.3 & 29 & 24 & 30 & 53 & 54 & 95 \\
200 & 25 & 0.3 & 51 & 41 & 85 & 38 & 38 & 72 \\
100 & 50 & 0.1 & 51 & 40 & 62 & 60 & 45 & 56 \\
150 & 50 & 0.1 & 102 & 81 & 124 & 114 & 92 & 111 \\
200 & 50 & 0.1 & 103 & 96 & 80 & 118 & 87 & 105 \\
150 & 100 & 0.1 & 260 & 179 & 341 & 292 & 207 & 407 \\
200 & 100 & 0.1 & 261 & 166 & 311 & 397 & 311 & 494 \\
200 & 150 & 0.1 & 454 & 308 & 516 & 430 & 264 & 536 \\
\hline
\end{tabular}

LP: $\quad$ Simplex method with least-reduced-cost rule

PDO: Primal and dual algorithm (original)

PDV: Primal and dual algorithm (variational)

Table 1: Iteration numbers for three pivot rules.

\section{Conclusions}

The preliminary computational tests suggest that our original primal and dual algorithm (PDO) performs increasingly well as the problem size increases, compared to the traditional simplex method (LP). They also suggest that PDO is generally better than the variational algorithm (PDV), though other test problems may reveal different performances.

We believe that further computational tests are justified for both new algorithms. 


\section{References}

[Dan63] G. B. Dantzig (1963). Linear Programming and Extensions, Princeton University Press, Princeton, NJ.

[Lem65] C. E. Lemke (1965). Bimatrix equilibrium points and mathematical programming, Management Science 11, 681-689.

[MLB87] C. Moler, J. Little and S. Bangert (1987). PRO-MATLAB User's Guide, The MathWorks, Inc., Sherborn, MA.

[TZ92] T. Terlaky and S. Zhang (1992). A survey of pivot rules for linear programming, Research Memorandum No. 480, Faculty of Economics, University of Groningen, The Netherlands. 

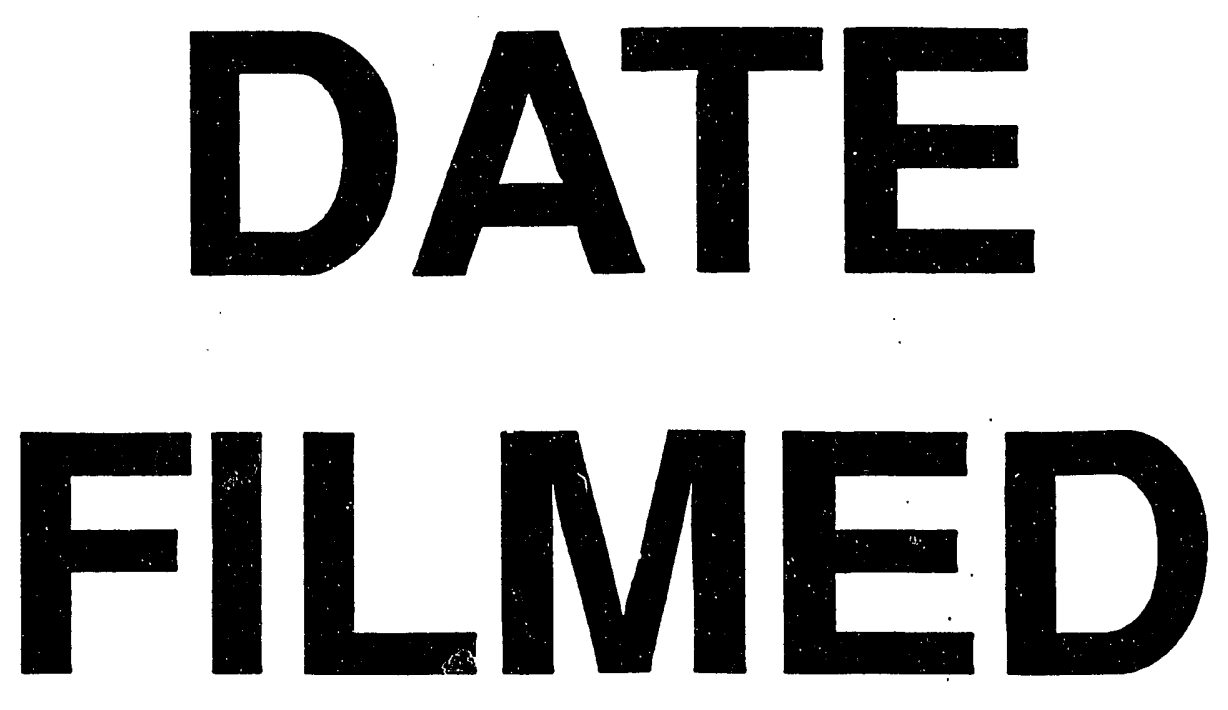

$10 / 13 / 93$
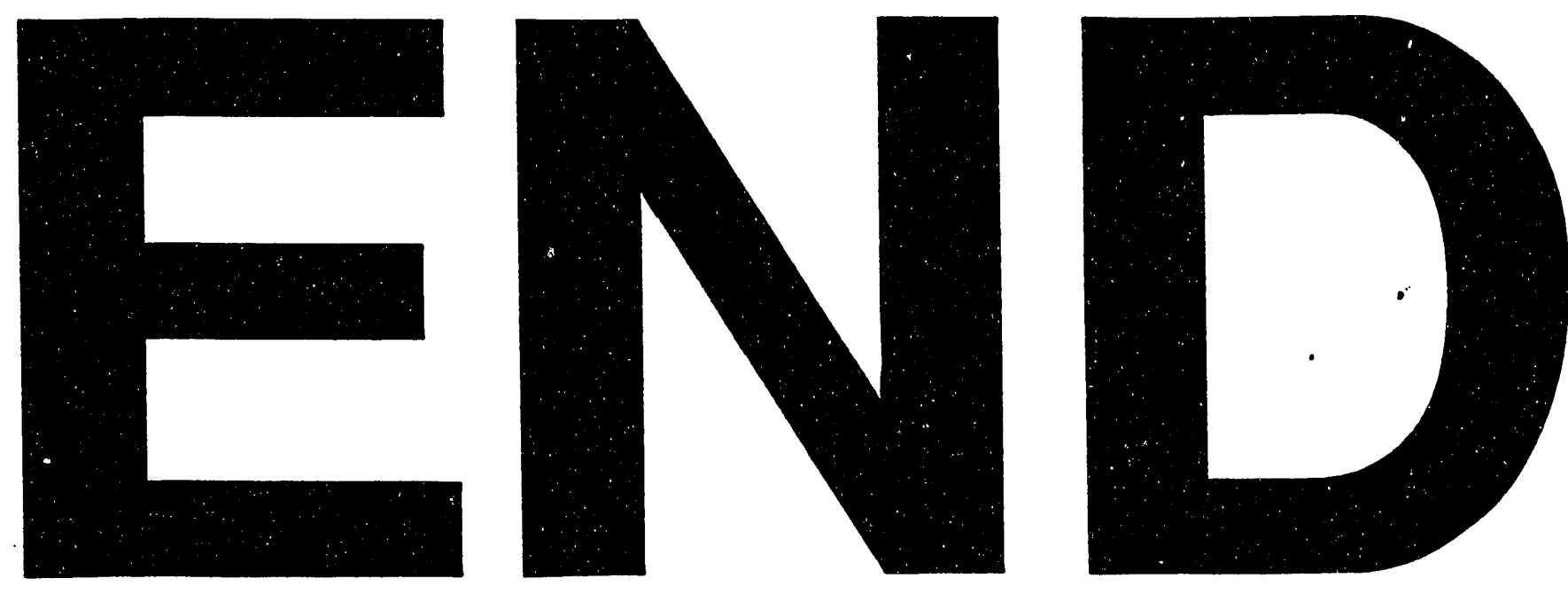
